\title{
Confinement Effects of Ion Tracks in Ultrathin Polymer Films
}

\author{
R. M. Papaléo, ${ }^{1, *}$ R. Thomaz, ${ }^{1}$ L. I. Gutierres,${ }^{1}$ V. M. de Menezes, ${ }^{2}$ D. Severin, ${ }^{3}$ C. Trautmann,,${ }^{3,4}$ \\ D. Tramontina, ${ }^{5}$ E. M. Bringa ${ }^{5}$ and P. L. Grande ${ }^{6}$ \\ ${ }^{1}$ Faculdade de Física, Pontifícia Universidade Católica do Rio Grande do Sul, Avenida Ipiranga 6681, 90619-900 Porto Alegre, Brazil \\ ${ }^{2}$ Campus Laranjeiras do Sul, Universidade Federal da Fronteira Sul, BR 158 km 405, 85301-970 Laranjeiras do Sul, Brazil \\ ${ }^{3}$ Materials Research, GSI Helmholtz Centre, Planckstrasse 1, 64291 Darmstadt, Germany \\ ${ }^{4}$ Technische Universität Darmstadt, Alarich-Weiss-Straße2, 64287 Darmstadt, Germany \\ ${ }^{5}$ Facultad de Ciencias Exactas y Naturales, Universidad Nacional de Cuyo, Mendoza 5500, Argentina \\ ${ }^{6}$ Instituto de Física, Universidade Federal do Rio Grande do Sul, Avenida Bento Gonçalves 9500, 91501-970 Porto Alegre, Brazil
}

(Received 21 November 2014; published 19 March 2015)

\begin{abstract}
We show direct experimental evidence that radiation effects produced by single MeV heavy ions on a polymer surface are weakened when the length of the ion track in the material is confined into layers of a few tens of nanometers. Deviation from the bulk (thick film) behavior of ion-induced craters starts at a critical thickness as large as $\sim 40 \mathrm{~nm}$, due to suppression of long-range additive effects of excited atoms along the track. Good agreement was found between the experimental results, molecular dynamic simulations, and an analytical model.
\end{abstract}

DOI: 10.1103/PhysRevLett.114.118302

PACS numbers: 79.20.Rf, 61.80.Jh, 61.82.Pv, 82.35.Lr

Energetic charged particles have been extensively used to characterize and modify materials in a controlled way, leading to a large number of important applications encompassing, among others, microelectronics, radiotherapy treatment, geological dating, and engineering of nanomaterials [1-4]. The extreme levels of energy deposited via electronic excitation (reaching up to several $\mathrm{keV} / \mathrm{nm}$ ) along narrow $(\sim 1-10 \mathrm{~nm})$ tracks are the basis of all unique radiation effects produced by high-energy ions in matter, including bond breaking [5], creation of defects [2], sputtering [6,7], and cratering [8,9]. Because of that, ion track formation and damage production by individual ions has been a central and persistent topic of research in radiation physics for decades [2,3]. Recently, with the growing use of nanostructured systems in various types of technological devices, the understanding of specificities in energy deposition and damage production by radiation in matter at the nanoscale has become a critical issue. Unique effects of energetic ions on nanoscale systems have already been reported and successfully applied to engineering of materials [10-13]. Nevertheless, very little has been reported so far on a direct comparison of radiation effects of heavy ions in a material at bulk and spatially confined conditions.

Here, polymer thin films have been used as a model system to probe radiation effects of individual $\mathrm{MeV}-\mathrm{GeV}$ ion tracks confined in one dimension, following the changes in surface effects (cratering and plastic deformation) as the thickness of the films is systematically reduced. We provide direct experimental evidence that radiation effects at the surface are weakened when the length of the ion track in the material is below critical dimensions as large as $\sim 40 \mathrm{~nm}$. We argue that this reduction of the radiation "strength" upon confinement arises essentially due to suppression of long-range additive effects of excited atoms along the track.

Thin films of poly(methyl methacrylate) (PMMA, Polymer Standards, Mw $132000 \mathrm{u}$ and $370000 \mathrm{u}$ ) of thicknesses $h$ varying from $\sim 1 \mathrm{~nm}$ up to $100 \mathrm{~nm}$ were spin coated from anisole solutions onto $\mathrm{Si}$ substrates (with a $2.3 \mathrm{~nm}$ native oxide layer). The samples were irradiated at the UNILAC accelerator at GSI, Darmstadt, by $923 \mathrm{MeV}$ $\mathrm{Pb}^{\mathrm{eq}+}$ and $2187 \mathrm{MeV} \mathrm{Au}^{\mathrm{eq}+}$ beams ( $\mathrm{eq}^{+}$indicates that the charge state of the ions had a distribution around equilibrium). Data obtained previously from samples irradiated with $597 \mathrm{MeV} \mathrm{Au}{ }^{45+}$ were also used for comparison [9]. Ion impact densities were $\sim 10$ ions $/ \mu \mathrm{m}^{2}$ and the angles of incidence $\theta$ were set at $0^{\circ}$ and $79^{\circ}$ with respect to the surface normal. The ion impact features were characterized off-line in air by means of scanning force microscopy (SFM) in the intermittent contact mode at a scan frequency of $1-2 \mathrm{~Hz}$ and 512 scan lines per image. Only images collected with high-quality $\mathrm{Si}$ tips were stored and used for quantitative analysis. Special care was taken of using the same tip (or tips of similar quality) to image a set of samples covering all thicknesses. A detailed study on the influence of the tip on the metrology of surface tracks is published in Ref. [14].

Figure 1 shows representative SFM images of ion impact features on films of different thicknesses at normal incidence [Figs. 1(a)-1(e), $923 \mathrm{MeV} \mathrm{Pb}^{\mathrm{eq}+}$ ] and at grazing incidence [Figs. 1(f)-1(j), $2.2 \mathrm{GeV} \mathrm{Au}{ }^{\mathrm{eq}+}$ ]. The electronic stopping power of both ions is around $14-16 \mathrm{keV} / \mathrm{nm}$. For the thicker layers $(h>40 \mathrm{~nm})$, a nanometer-sized crater surrounded by a rim is formed at the point of ion impact [Fig. 1(a)]. Under grazing beam incidence, elongated craters and protrusions appear along the direction of ion penetration [Fig. 1(f)]. These are typical surface features 


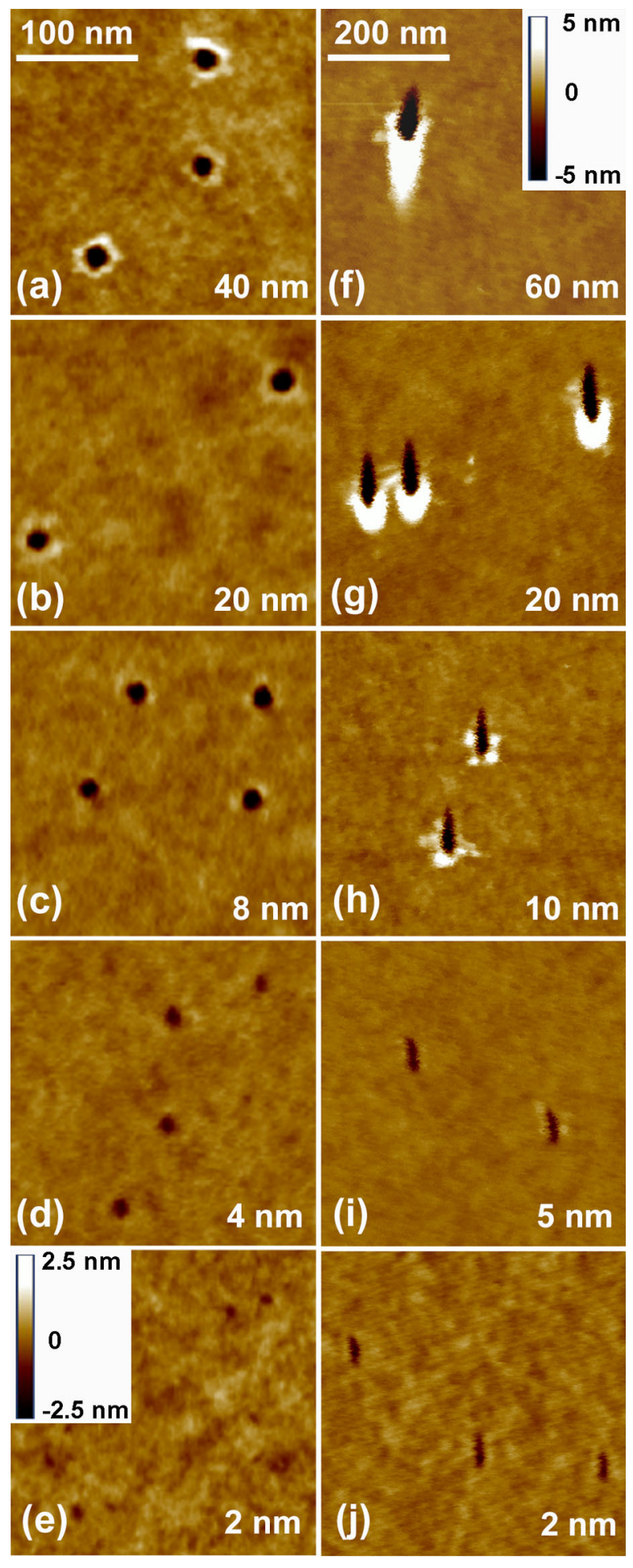

FIG. 1 (color online). SFM images of PMMA thin films of different thicknesses bombarded by (a)-(e) $923 \mathrm{MeV} \mathrm{Pb}^{\text {eq }}+$ ions at normal incidence and by (f)-(j) $2.2 \mathrm{GeV} \mathrm{Au}{ }^{\text {eq+ }}$ ions at grazing incidence. The thickness of the layer is given in each image. The scale bar of $100 \mathrm{~nm}$ is for images (a)-(e) and the $200 \mathrm{~nm}$ bar is for images (f)-(j). The height scale given in (f) is valid for all images, except in (e). seen on polymers $[9,14]$ and other organic materials [15] due to massive mass transport and particle ejection emerging from the core of $\mathrm{MeV}-\mathrm{GeV}$ ion tracks. As the morphology and size of the surface features do not change with further increase in target thickness, this is what we call the "bulk" condition for this particular radiation effect. For films of thickness below $\sim 40 \mathrm{~nm}$, rims and tails quickly diminish in size. Craters also start to become slightly smaller but are less affected by the thickness reduction. At $h<10 \mathrm{~nm}$, craters are still clearly observed, but protrusions have been completely suppressed.

Figure 2 depicts quantitative data on averaged sizes of craters and rims as a function of layer thickness $h$ for tracks produced by $923 \mathrm{MeV} \mathrm{Pb}$ and $597 \mathrm{MeV} \mathrm{Au}$ at normal incidence (solid symbols). All crater dimensions present a similar trend: saturation at large thicknesses and decrease in size for $h$ below a certain critical value $h_{c}$. However, $h_{c}$ is not unique, but depends on the type of effect: $h_{c} \sim 40 \mathrm{~nm}$ for the rim volume [Fig. 2(c)] and $h_{c} \sim 10 \mathrm{~nm}$ for the crater diameter [Fig. 2(a)]. We note that the decrease in the dimensions of the impact features with thickness for $h<h_{c}$ is not simply a matter of lack of material to be displaced or ejected in the ultrathin layers. For example, rim size starts to decrease well before a thickness comparable to the height of the protrusion at bulk conditions $(\sim 2 \mathrm{~nm})$ is reached.

The formation of craters and protrusions due to a fast ion impact is usually attributed to local heating and/or to mechanical disturbances originating from the excited track core. In pure thermal models (as in the analytical thermal spike model), it is the energy density at the surface that controls particle ejection and cratering or hillock formation $[7,19]$. MD simulations have also shown the importance of coherent movement of a volume of atoms (pressure waves), in addition to local heating, at high excitation densities $[20,21]$. Such correlated momentum transfer seen on simulations and experiments with organic materials [6] has been analytically described by expansion models like the pressure pulse model [22]. In this model, atoms are set in motion by momentum transport from the excited track core region. The resultant impulse imparted to a volume of material at the surface is the sum of "mini" perturbations, each produced at and propagating diffusively from an element $d z$ along the ion track. Such an approach provides a suitable and simple theoretical framework to apply to our experimental observations, because it allows us to estimate how the source of energy and momentum changes as the thickness of the layer $h$ decreases.

The momentum imparted to an element of volume of the material at a radial distance $\rho$ and depth $z$ is

$$
p(\rho, z, h)=\frac{\beta}{n_{M}} \int_{0}^{\infty}-\nabla \epsilon(\rho, z, t, h) d t,
$$




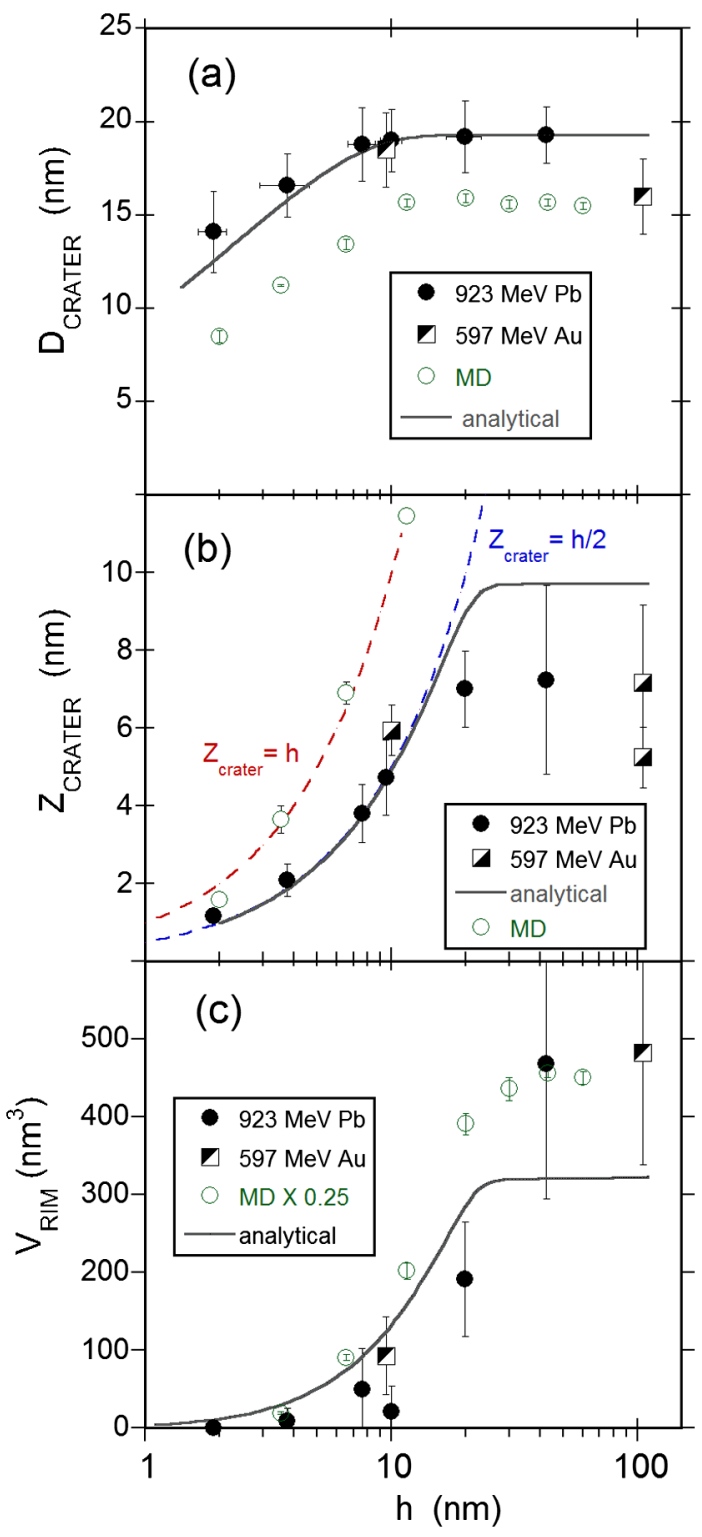

FIG. 2 (color online). Averaged crater dimensions produced by single heavy ions on PMMA as a function of film thickness $h$ for normal incidence: (a) crater diameter, (b) crater depth, and (c) volume of the protruded rim above the unperturbed surface position. In (b) a data point for $h=100 \mathrm{~nm}$ measured with a blunt tip was also included. Solid lines show the results for the analytical model and open circles are simulation data (details in Supplemental Material [16]).

where $n_{M}$ is the molecular number density. $\beta$ is a materialdependent constant related to the fraction of energy gained by the molecules which appears as translational energy [22]. The procedure to estimate the time-dependent total deposited energy density $\epsilon(\rho, z, t, h)$ from the addition of all point sources along the track is described in detail in Refs. [20,22] and outlined in the Supplemental Material [16]. An example of 2D maps of the normal component of the transferred momentum $p_{z}(\rho, z, h)$ for a thick and a thin film is given in Fig. S1 of the Supplemental
Material [16]. Assuming that the crater is formed from the ejection of particles receiving $p_{z}$ larger than a critical value $p_{c}^{C}$, the crater radius and depth as a function of $h$ can be readily extracted from the maps and compared to experimental results. Below, we present the overall qualitative behavior of the model, using the onset temperature for unzipping of PMMA chains into volatile monomer units $\left(T_{c}=493 \mathrm{~K}\right)$ to estimate the critical momentum for ejection $\left(p_{c}^{C} \cong \sqrt{3 M k T_{c}}\right.$ ). Other input parameters (such as diffusivity $\kappa$ and the rate of energy dissipation $1 / \tau$ ) and their effect on the calculations are presented in the Supplemental Material [16].

Curves for the crater diameter as a function of $h$ are shown as solid lines in Fig. 2(a). Despite the simplicity of the analytical approach, the match between the calculations based on the linear addition of energy sources and the experimental data is surprisingly good. The analytical model also predicts correctly the variation of the crater depth $Z_{\text {crater }}$ with $h$ seen experimentally [Fig. 2(b)]. For thick samples, the predicted crater profile is a half-sphere with $Z_{\text {crater }}=D_{\text {crater }} / 2$ [Fig. S1(a) of the Supplemental Material [16]]. Thus, the depth of the crater at bulk conditions $Z_{\text {crater }}^{\text {sat }}$ equal to the crater radius, similarly to what is seen experimentally $\left(D_{\text {crater }} / 2 \sim Z_{\text {crater }}^{\text {sat }} \sim 8-10 \mathrm{~nm}\right)$. Because $Z_{\text {crater }}$ decreases much faster with $h$ than $D_{\text {crater }}$, the predicted crater profiles change from a half-sphere to a shallow bowl for ultrathin films. Moreover, in the analytical model $Z_{\text {crater }}$ is at most $h / 2$ : the net momentum points towards the surface only at points in the film down to $h / 2$. Below $h / 2$ the net momentum reverses, pushing the molecules towards the substrate [Fig. S1(b) of the Supplemental Material [16]]. Thus, for layers thinner than $h \sim 2 Z_{\text {crater }}^{\text {sat }}(\sim 20 \mathrm{~nm}$ in the experiments), the crater depth is expected to evolve as $Z_{\text {crater }}=h / 2$. This is in striking agreement with the experiments, as can be seen in Fig. 2(b), considering that the actual transport of mass is not included in the analytical description. For instance, melt flow, which might increase crater depth [23], and relaxation of the hot crater walls, which may decrease the final depth, are not included.

The volume of the crater rim shown in Fig. 2(c) is more difficult to directly link to the impulse maps. A crude comparison can be made assuming that the displacement yield is proportional to the volume of the material receiving an impulse $p_{c}^{R}$ enough for plastically displacing a molecule, but insufficient for ejection, i.e., by estimating the volume of material with $p_{c}^{R}<p_{z}<p_{c}^{C}$. The volume of molecules satisfying this, with $p_{c}^{R}=0.75 p_{c}^{C}$, is shown as a solid line in Fig. 2(c). Again, the overall dependence of the rim volume with $h$ is well reproduced by the sum of impulses approach.

In order to get complementary information to the analytical model, we have carried out MD simulations using LAMMPS [24] and a cylindrical thermal spike to model the ion track. Simulations are similar to the ones used to explain scaling of crater features in polymers using 
a Lennard-Jones potential with $\epsilon=0.1 \mathrm{eV}$ and $\sigma=0.5 \mathrm{~nm}$ [21], but here we use an amorphous medium with several million particles, to better match experimental samples. Using more complex potentials to describe such polymer samples is beyond our computational resources. The ion track is limited to a depth $h$, while the sample below is assumed to behave as a substrate where excitation can be neglected (see Supplemental Material for details [16]).

Figure S3 of Ref. [16] displays a surface image from MD similar to SFM pictures. Figure 3 shows the final snapshot from one MD simulation, including displacement vectors from initial positions, with upward arrows starting near $z \sim h / 2$ in agreement with pressure pulse predictions. Below that depth, displacements have a high degree of randomness, or tend to point downwards. There is clear evidence for significant collective motion towards the surface, due to the momentum transfer caused by the pressure pulse in the track. This is remarkable, because collision sequences in amorphous media tend to be short, unlike what happens in crystals [25]. MD craters and rims [open circles in Figs. 2(a)-2(c)] show significant decrease with $h$ and at similar critical lengths, as in the experiments. Crater diameters are comparable to experimental ones, being mostly determined by ejection. Melt flow, however, indicated in Fig. 3 by large displacement arrows originating from the inner regions of the track, contributes to increasing crater depth and rim volume. In the MD simulations, the depth of the craters follows roughly the curve $Z_{\text {crater }}=h$, but ejected particles originate mostly from $z<h / 2$ (inset of Fig. 3), as in the analytical model. Entangled polymers will have much higher viscosity than our simplified MD model, and therefore it is reasonable that a quantitative

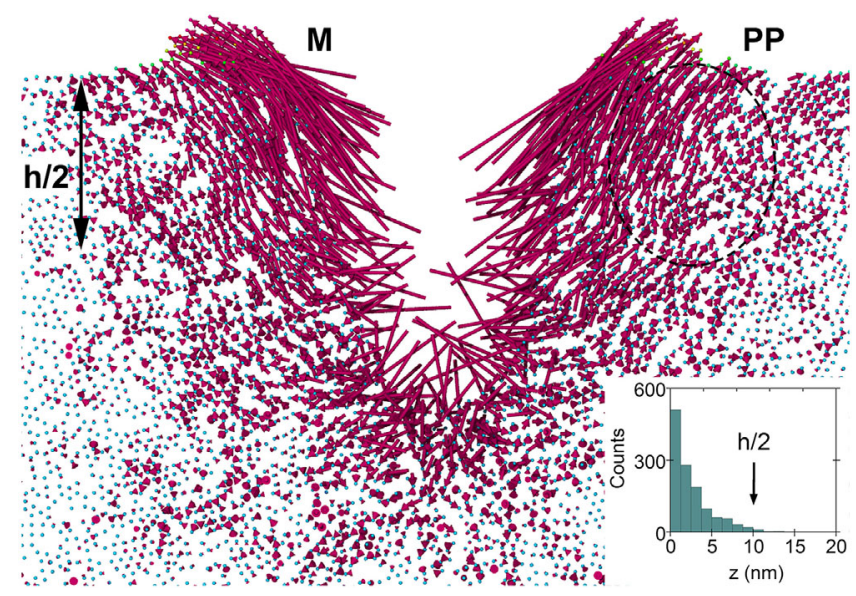

FIG. 3 (color online). Final snapshot $(t=160 \mathrm{ps})$ from one MD simulation $(h=20 \mathrm{~nm})$, including displacement vectors from initial positions. Only a thin slice through the center of the crater is shown. PP indicates collective displacements due to the pressure pulse and $\mathrm{M}$ the melt flow contributing to the rim volume. The inset shows the number of ejected particles as a function of depth $(98 \%$ comes from $z<h / 2)$. disagreement is found, even if the qualitative confinement effect is observed.

In summary, we demonstrate that radiation effects at the surface, produced by ion tracks in a polymer, are weakened when the relevant electronic excitations are spatially confined into layers with a thickness below few tens of nanometers. This is in contrast to experiments performed in other 2D inorganic insulators, where an enhancement of the track "strength" (i.e., either longer track lifetime or higher track temperatures) was deduced [26,27]. We argue that the confinement effects seen here arise essentially due to the decrease of the number of point sources of excitation energy in shorter track lengths. Effects that strongly depend on the cooperative action of energy sources along the track (as rim formation) are suppressed first, resulting in large critical lengths. This is most likely a general feature in weakly bound insulators for which electronic excitations have an efficient, fast coupling to the atomic cores. Materials with different diffusivity and dissipation rate would only change the critical length at which confinement effects start. We also provide strong evidence for the importance of momentum transfer, which limits the depth of origin of ejected particles to a maximum of $h / 2$, even at the very thin layers and large $d E / d x$ used in the experiments. Correlated momentum transfer may also operate in rigid, inorganic insulators, but with less evident effects due to the much higher binding energy in those materials.

Here we addressed confinement effects directly related to particle transport and ejection induced by dense electronic excitations. Our findings have practical significance to dosimetry of ion beams in small volumes, to radiation strength of organic nanomaterials, and for devices set in the space environment, particularly those involving organic electronics. An important open question is whether spatial confinement alters the coupling of the electronic excitation to atomic motion, or radiation chemistry induced by heavy ions in organic matter, and at what critical length. Additional energy dissipation through the underlying substrate, e.g., via exciton diffusion or heat transfer, may also be important. This is yet to be tested experimentally by varying the type of substrate.

The agencies FAPERGS, CAPES, and CNPq (Brazil), DAAD (Germany), and CONICET (Argentina) are acknowledged for financial support.

* Corresponding author. papaleo@pucrs.br

[1] J. R. Tesmer and M. Nastasi, Handbook of Modern Ion Beam Materials Analysis (Materials Research Society, Pittsburgh, 1995).

[2] R. L. Fleischer, P. Price, and R. M. Walker, Nuclear Tracks in Solids (University of California Press, Berkeley, 1975). 
[3] R. Hellborg, H. Whitlow, and Y. Zhang, Ion Beams in Nanoscience and Technology (Springer-Verlag, Berlin, 2009).

[4] T. Kanai et al., Int. J. Radiat. Oncol. Biol. Phys. 44, 201 (1999).

[5] T. Venkatesan, S. R. Forrest, M. L. Kaplan, P. H. Schmidt, C. A. Murray, W. L. Brown, B. J. Wilkens, R. F. Roberts, L. Rupp, and H. Schonhorn, J. Appl. Phys. 56, 2778 (1984).

[6] W. Ens, B. U. R. Sundqvist, P. Hakansson, A. Hedin, and G. Jonsson, Phys. Rev. B 39, 763 (1989).

[7] M. Toulemonde, W. Assmann, C. Trautmann, and F. Gruner, Phys. Rev. Lett. 88, 057602 (2002).

[8] F. Thibaudau, J. Cousty, E. Balanzat, and S. Bouffard, Phys. Rev. Lett. 67, 1582 (1991).

[9] R. M. Papaléo, M. R. Silva, R. Leal, P. L. Grande, M. Roth, B. Schattat, and G. Schiwietz, Phys. Rev. Lett. 101, 167601 (2008).

[10] H. Bernas, J. P. Attane, K. H. Heinig, D. Halley, D. Ravelosona, A. Marty, P. Auric, C. Chappert, and Y. Samson, Phys. Rev. Lett. 91, 077203 (2003).

[11] E. Akcoltekin, T. Peters, R. Meyer, A. Duvenbeck, M. Klusmann, I. Monnet, H. Lebius, and M. Schleberger, Nat. Nanotechnol. 2, 290 (2007).

[12] G. Rizza et al., Phys. Rev. B 86, 035450 (2012).

[13] E. M. Bringa et al., Nano Lett. 12, 3351 (2012).

[14] L. S. Farenzena, R. P. Livi, M. A. de Araujo, G. G. Bermudez, and R. M. Papaléo, Phys. Rev. B 63, 104108 (2001).
[15] J. Eriksson, J. Rottler, C. T. Reimann, and B. U. R. Sundqvist, Int. J. Mass Spectrom. Ion Process. 175, 293 (1998).

[16] See Supplemental Material at http://link.aps.org/ supplemental/10.1103/PhysRevLett.114.118302, which includes Refs. $[17,18]$, for technical details on the analytical model and molecular dynamics simulations.

[17] M. Backman, F. Djurabekova, O. H. Pakarinen, K. Nordlund, Y. Zhang, M. Toulemonde, and W. J. Weber, Nucl. Instrum. Methods Phys. Res., Sect. B 303, 129 (2013).

[18] D. Schwen, E. Bringa, J. Krauser, A. Weidinger, C. Trautmann, and H. Hofsass, Appl. Phys. Lett. 101, 113115 (2012).

[19] R. R. Lucchese, J. Chem. Phys. 86, 443 (1987).

[20] D. Fenyö and R. E. Johnson, Phys. Rev. B 46, 5090 (1992).

[21] E. M. Bringa, R. E. Johnson, and R. M. Papaléo, Phys. Rev. B 65, 094113 (2002).

[22] R. E. Johnson, B. U. R. Sundqvist, A. Hedin, and D. Fenyö, Phys. Rev. B 40, 49 (1989).

[23] C. Anders, G. Ziegenhain, C. J. Ruestes, E. M. Bringa, and H. M. Urbassek, New J. Phys. 14, 083016 (2012).

[24] S. Plimpton, J. Comput. Phys. 117, 1 (1995).

[25] K. Nordlund, J. Keinonen, M. Ghaly, and R. S. Averback, Nature (London) 398, 49 (1999).

[26] P. I. Gaiduk and C. Trautmann, Nucl. Instrum. Methods Phys. Res., Sect. B 256, 224 (2007).

[27] R. K. Pandey, M. Kumar, S. A. Khan, T. Kumar, A. Tripathi, D. K. Avasthi, and A. C. Pandey, Appl. Surf. Sci. 289, 77 (2014). 